\title{
Slow, repetitive transcranial magnetic stimulation was effective for major depression
}

\author{
Klein E, Kreinin I, Chistyakov A, et al. Therapeutic effect of right prefrontal slow repetitive transcranial magnetic stimulation in \\ major depression. A double-blind controlled study. Arch Gen Psychiatry 1999 Apr;56:315-20.
}

\section{Question}

In patients with major depression, is slow, repetitive transcranial magnetic stimulation (rTMS) effective for relieving depressive symptoms?

\section{Design}

2 week randomised, double blind, placebo controlled trial.

\section{Setting}

$\{$ Psychiatric department of a general hospital $\} *$ in Israel.

\section{Patients}

70 patients (mean age 59 y, 76\% women) who had major depression according to the DSM-IV; were right handed; had normal neurological and general physical examination results; did not have a history of major brain trauma, seizure disorder, or substance abuse; and were not medication resistant. Follow up was $96 \%$.

\section{Intervention}

Patients were allocated to rTMS $(n=36)$ or sham rTMS $(n=34)$. In rTMS, a magnetic stimulator (Cadwell Inc, Kennewick, Washington, USA) with a $9 \mathrm{~cm}$ external diameter circular coil was placed over the right prefrontal area $6 \mathrm{~cm}$ anterior to the scalp position at which the motor threshold was determined. In sham rTMS, the stimulation coil was placed perpendicular to the scalp without direct contact. 10 daily sessions were given over 2 weeks.

\section{Main outcome measures}

Severity of depression (assessed with the Hamilton Depression Rating Scale [HDRS] and the Montgomery-Asberg Depression Rating Scale [MADRS]) at baseline, after 1 week of treatment, and at 24 hours after the last treatment session.

\section{Main results}

More patients in the rTMS group than in the sham-rTMS group had $\geq 50 \%$ reduction in HDRS or MADRS scores $(\mathrm{p}<0.05)$ and had a final HDRS score $\leq 10(\mathrm{p}<0.02)$ (table). No serious adverse effects were reported in either group.

\section{Conclusion}

In patients with major depression, slow, repetitive transcranial magnetic stimulation led to a reduction in depressive symptoms.

*Information provided by author.

Slow, repetitive transcranial magnetic stimulation ( $r$ TMS) v sham rTMS in major depressiont

\begin{tabular}{lllll}
\hline Outcomes at 2 weeks & RTMS & $\begin{array}{l}\text { Sham } \\
\text { rTMS }\end{array}$ & RBI (95\% CI) & NNT (CI) \\
\hline $\begin{array}{l}\text { 250\% reduction in } \\
\text { HDRS or }\end{array}$ & & & & \\
MADRS scores & $49 \%$ & $25 \%$ & $94 \%(1$ to 293$)$ & 5 (3 to 466$)$ \\
HDRS score $\geqslant 10$ & $46 \%$ & $19 \%$ & $144 \%(15$ to 450$)$ & $4(3$ to 23$)$ \\
\hline
\end{tabular}

†HDRS $=$ Hamilton Depression Rating Scale; $M A D R S=$ Montgomery-Asberg Depression Rating Scale. Other abbreviations defined in glossary; RBI, NNT, and CI calculated from data in article.

\section{Source of funding: Stanley Foundation.}

For correspondence: Dr E Klein, Department of Psychiatry, Rambam Medical Center, Efron Street, Bat-Galim, Haifa 31096, Israel. Fax +97248514285.

Abstract and commentary also published in Evidence-Based Medicine.

\section{Commentary}

Rapid rTMS is a method of stimulating the cerebral cortex by using magnetic fields generated in an electrical coil. Applied to appropriate brain areas, rTMS can influence cognitive function and perhaps mood in healthy people. The abnormalities in prefrontal perfusion found by functional brain imaging studies in some depressed patients have led to therapeutic trials of rTMS in major depression.

Klein et al found clinically significant antidepressant effects with low frequency rTMS applied to the right prefrontal area. This study has substantial methodological differences from other positive controlled studies of rTMS in regard to the stimuli used, their site of application, and the coil used to deliver them. ${ }^{1}$ The level of severity of the patients' symptoms is not easy to assess. On the one hand, they were inpatients, but, on the other, none was deemed treatment resistant. Such patients would be unusual in an inpatient setting in the UK.

Antidepressant effects of rTMS raise the tantalising prospect that it may be possible to replace electroconvulsive therapy (ECT) with a treatment that does not require anaesthesia or cause fits or cognitive impairment. Unlike most patients treated with ECT, however, patients in this study were not medication resistant, and concomitant medication may have influenced the therapeutic response to rTMS.

More work is required to define the most appropriate stimulus variables and placement for rTMS in the treatment of depression. In addition, a more convincing placebo treatment than sham application needs to be devised. The failure of some studies to replicate specific antide- pressant effects of rTMS suggests that this is not a trivial issue. ${ }^{12}$ Another important area for study is the duration of therapeutic effect of rTMS. The successful use of ECT in medication resistant patients is unfortunately often followed by high relapse rates and, presumably, similar problems might attend the use of rTMS in such patients.

\section{Philip J Cowen, MD University of Oxford Oxford, UK}

1 George MS, Lisanby SH, Sackeim HA. Transcranial magnetic stimulation: applications in 1999;56:300-11. Arch Gen Psychiatry

2 Loo C, Mitchell P, Sachdev P, et al. Doubleblind controlled investigation of transcranial magnetic stimulation for the treatment of resistant major depression. Am J Psychiatry
1999;56:946-8. 